\title{
Transtorno do pânico
}

\author{
Panic disorder
}

\section{Giovanni Abrahão Salum¹, Carolina Blaya ${ }^{2}$, Gisele Gus Manfro ${ }^{3}$}

${ }^{1}$ Bolsista, Iniciação Científica, Conselho Nacional de Desenvolvimento Científico e Tecnológico (CNPq). Pesquisador, Programa de Transtornos de Ansiedade na Infầncia e Adolescência, Hospital de Clínicas de Porto Alegre (HCPA), Porto Alegre, RS. ${ }^{2}$ Mestre, Doutora, Ciências Médicas, Psiquiatria, Universidade Federal do Rio Grande do Sul (UFRGS), Porto Alegre, RS. Pesquisadora, Programa de Transtornos de Ansiedade, HCPA. ${ }^{3}$ Professora adjunta, Departamento de Psiquiatria e Medicina Legal, UFRGS. Coordenadora, Programa de Transtornos de Ansiedade e Programa de Transtornos de Ansiedade na Infância e Adolescência, HCPA.

\section{Resumo}

Objetivos: Revisar o transtorno do pânico (TP), considerando seus aspectos clínicos, epidemiológicos, diagnósticos e etiológicos, bem como os avanços no tratamento, uma vez que o TP é uma entidade nosológica acompanhada de importante prejuízo psíquico e funcional.

Método: Foi realizada uma revisão narrativa da literatura nas principais bases de dados existentes (MEDLINE, PsychINFO e SciELO) e em livrostextos atualizados.

Resultados: Devido à sintomatologia predominantemente física desse transtorno, os pacientes geralmente procuram vários atendimentos clínicos até que o diagnóstico seja feito. Em função desses aspectos e da sua cronicidade, o TP está associado a elevados custos econômicos. O tratamento do TP pode ser feito com psicoterapia e/ou psicofármacos. As diversas abordagens terapêuticas são apresentadas com o nível de evidência de cada recomendação. Em virtude da cronicidade e morbidade do TP, pesquisas têm se voltado para o estudo de estratégias de prevenção já na infância. Conclusões: O TP é um transtorno crônico e com baixas taxas de remissão dos sintomas em longo prazo. Sendo assim, sugere-se que sejam delineados novos estudos para tratamento precoce dos transtornos de ansiedade ou mesmo para prevenção em crianças de risco.

Descritores: Transtorno do pânico, transtornos de ansiedade, diagnóstico, tratamento, farmacoterapia, psicoterapia.

\section{Abstract}

Objective: To review panic disorder (PD) considering its clinical, epidemiological, diagnostic, and etiologic aspects, as well as the advances in its treatment, since PD is a nosologic entity characterized by important psychiatric and functional impairment.

Method: A review of the literature was carried out using the main databases available (MEDLINE, PsychINFO and SciELO) and up-to-date textbooks.

Results: Due to the prevalence of physical symptoms in this disorder, patients usually have to seek clinical care for several times before the diagnosis is established. As a result of these aspects and because of its chronicity, PD is associated with high economic costs. PD treatment may include psychotherapy and/or use of psychotropic drugs. In this review, we present several different therapeutic approaches, as well as the levels of evidence of each recommendation. Due to PD chronicity and morbidity, researches have been focused on the study of strategies to prevent PD since childhood.

Conclusions: PD is a chronic disorder with low long-term remission rates. Therefore, we suggest that new studies should be designed concerning the early treatment of anxiety disorders or even the prevention of this disorder in children at risk.

Keywords: Panic disorder, anxiety disorder, diagnosis, treatment, pharmacotherapy, psychotherapy.

\section{Correspondência:}

Gisele Gus Manfro, Hospital de Clínicas de Porto Alegre, Ramiro Barcelos 2350, sala 400N, CEP 90035-003, Porto Alegre, RS. E-mail: gmanfro@hcpa.ufrgs.br Não há conflitos de interesse associados à publicação deste artigo.

Copyright (C) Revista de Psiquiatria do Rio Grande do Sul - APRS 


\section{Introdução}

O transtorno do pânico (TP) é caracterizado pela presença de ataques de pânico recorrentes que consistem em uma sensação de medo ou mal-estar intenso acompanhada de sintomas físicos e cognitivos e que se iniciam de forma brusca, alcançando intensidade máxima em até 10 minutos. Estes ataques acarretam preocupações persistentes ou modificações importantes de comportamento em relação à possibilidade de ocorrência de novos ataques de ansiedade.

Os pacientes com TP seguem um padrão longo (que pode se estender a até uma década) de visitas às emergências médicas antes do diagnóstico à procura de uma causa orgânica para seus sintomas ${ }^{1}$. Portanto, além de psiquiatras, também médicos em geral e, em especial, aqueles que trabalham com atenção primária e serviços de emergência médica devem estar familiarizados com os critérios do TP. O conhecimento desses profissionais é de extrema importância ao considerarmos a alta prevalência dos ataques de pânico na população em geral e a necessidade de saber diferenciar os ataques de pânico isolados da síndrome completa (o TP) e de outros problemas médicos que podem se apresentar como uma crise de ansiedade.

Além do sofrimento psíquico e do prejuízo funcional vivenciados pelos pacientes com TP, ele está associado a uma série de outros desfechos que, empiricamente, justificam seu tratamento como um problema de saúde pública. Pacientes com TP têm maiores taxas de absenteísmo e menor produtividade no trabalho ${ }^{2}$; maiores taxas de utilização dos serviços de saúde, procedimentos e testes laboratoriais ${ }^{3}$; um risco aumentado, independente das comorbidades, de ideação de suicídio e de tentativas de suicídio ${ }^{4,5}$; e, em mulheres pósmenopáusicas, parece estar relacionado à morbidade e mortalidade cardiovasculares ${ }^{6}$. No entanto, cabe ressaltar que a associação com mortalidade cardiovascular ainda é controversa e pode se restringir a uma população específica ${ }^{7}$.

$\mathrm{O}$ presente artigo de atualização tem como objetivo revisar o TP, considerando seus aspectos clínicos, de prevalência, diagnóstico e etiologia, bem como os avanços no tratamento. Para tanto, foi realizada uma revisão narrativa da literatura nas principais bases de dados existentes (MEDLINE, PsychINFO e SciELO) e em livros-textos atualizados.

\section{Revisão narrativa}

Embora o TP tenha surgido como uma entidade diagnóstica há 9 anos com a publicação do Diagnostic and Statistical Manual of Mental Disorders (DSM) III $^{8}$, referências a essa entidade clínica apareceram muito antes disso (por exemplo, coração de soldado, de Da Costa9 ${ }^{9}$, astenia neurocirculatória, de Wheeler ${ }^{10}$, e síndrome de esforço, de Lewis $\left.{ }^{11}\right)$. Além da ativação paroxística do sistema nervoso autonômico (SNA) e das cognições catastróficas, essas síndromes envolviam sintomas de fadiga profunda que não fazem parte dos critérios diagnósticos atuais. De todos os transtornos ansiosos, o TP foi o transtorno mais estudado nos últimos 25 anos; no entanto, ainda existem lacunas importantes em termos de diagnóstico e classificação, etiologia e tratamento dessa condição clínica.

\section{Prevalência}

A prevalência dos ataques de pânico é muito comum. O National Comorbidity Survey (NCS) Replication ${ }^{12}$, envolvendo uma amostra representativa da população estadunidense, estimou que $23 \%$ dessa população preencheu critérios para ao menos um ataque de pânico isolado ao longo da vida. A prevalência do TP, contudo, é menos comum, já que, ao longo da vida, $5 \%$ (4\% sem agorafobia, e $1 \%$ com agorafobia) dos respondentes preencheram critérios para o transtorno, alcançando cerca de $1 \%$ no último ano. A prevalência de agorafobia com ataques de pânico sem preencher critérios para TP também foi de aproximadamente $1 \%$. A presença de agorafobia aumenta ainda mais a incapacidade e a gravidade clínica: mais de $95 \%$ dos entrevistados apresentaram incapacidades de moderadas a graves relacionadas ao transtorno, enquanto apenas $21 \%$ dos pacientes com ataques de pânico isolados sofreram incapacidades de moderadas a graves ${ }^{12}$.

Não existem dados com amostra representativa da população brasileira. Um estudo realizado na cidade de São Paulo (SP) encontrou uma prevalência de $1,6 \%$ para o TP ao longo da vida e de $1 \%$ em 1 ano $^{13}$. Não parece haver uma variação na prevalência ao redor do mundo, mas isso não se pode afirmar ao certo em função das diferentes abordagens metodológicas dos diversos estudos. O TP é cerca de duas vezes mais comum em mulheres e, geralmente, inicia no final da adolescência ou no início da vida adulta, com média de aparecimento entre os 20 e 30 anos; raramente, há casos de aparecimento na infância ${ }^{12,14}$.

A comorbidade psiquiátrica é regra no TP e não exceção. Quase 100\% dos pacientes com TP com agorafobia e cerca de $83 \%$ dos pacientes com TP sem agorafobia apresentam ao menos uma comorbidade psiquiátrica. Considerando o TP com agorafobia, $95 \%$ têm ao menos algum outro transtorno de ansiedade, $64 \%$ têm ao menos um transtorno de humor, $63 \%$ têm algum transtorno de controle de impulsos e 31\% apresentam quadros de abuso ou dependência de álcool e drogas. Dentre os transtornos de ansiedade, a fobia específica é a comorbidade mais comum (presente em $74 \%$ dos casos), seguida do transtorno de ansiedade social $(66 \%)$, do transtorno de ansiedade generalizada (32\%) e da ansiedade de separação (13\%). Dentre os transtornos do humor, quase $50 \%$ preenchem critérios para depressão maior, $16 \%$ para transtorno do humor bipolar (tipos I e II) e 10\% para distimia. Considerando dependência de álcool e drogas, $14 \%$ preencheram critérios para apenas dependência de álcool, e $11 \%$ para dependência de drogas ${ }^{12}$.

\section{Etiologia}

Diversos fatores vêm sendo atribuídos à etiologia do TP, sendo que tanto fatores genéticos quanto ambientais parecem contribuir para esse transtorno ${ }^{15}$. 
Os transtornos de ansiedade geralmente se agregam em famílias ${ }^{16}$. Estudos de famílias com TP demonstraram um padrão familiar, com um risco de TP, em parentes de primeiro grau dos pacientes com esse transtorno, cerca de oito vezes maior do que o do grupo-controle ${ }^{17}$. Estudos com gêmeos também corroboram esses achados, mostrando maior concordância do TP em gêmeos monozigóticos em comparação aos dizigóticos, com uma herdabilidade estimada de aproximadamente $43 \%{ }^{16}$.

Estudos prévios têm associado experiências traumáticas na infância ${ }^{18-20}$ ao desenvolvimento do TP na idade adulta. Eventos estressantes na vida adulta também estão relacionados ao desenvolvimento de transtornos de ansiedade ${ }^{21} \mathrm{e}$ ao $\mathrm{TP}^{22}$, sendo que cerca de $80 \%$ dos pacientes relataram estressores de vida nos 12 meses que precederam o início do transtorno. Scocco et al. ${ }^{23}$ descreveram que cerca de $90 \%$ dos pacientes com TP experienciaram transição de papéis e que $40 \%$ tiveram perdas no ano que antecedeu o início do TP. Fatores socioeconômicos como etnia, estado civil, grau de escolaridade e renda não parecem ser fatores de maior importância na associação com o $\mathrm{TP}^{12}$. O tabagismo e a dependência de nicotina na adolescência têm sido implicados como fatores de risco para o TP na vida adulta, mas a causalidade dessa associação ainda é questionável ${ }^{24}$.

Dentro dos transtornos de ansiedade e especificamente em relação ao TP, espera-se que genes tenham algum papel mais ligado à determinação de fenótipos intermediários, isto é, características que são comuns, em maior ou menor grau, a todos os indivíduos, e que estão associados a um ou mais transtornos psiquiátricos de forma específica ou inespecífica. Dentre os fenótipos intermediários dos transtornos de ansiedade na infância, pode-se citar o comportamento inibido (inibição do comportamento associado ao medo de pessoas não familiares) $\mathrm{e}$ a sensibilidade à ansiedade (medo e preocupações acerca de interpretações distorcidas de reações corporais normais). Na vida adulta, o neuroticismo (ansiedade, depressão, tensão, irracionalidade, emoção, baixa autoestima e tendência a sentimentos de culpa) e alguns temperamentos específicos como a evitação de danos (tendência a evitar situações com algum tipo de risco, ou que causem ou possam causar danos) estão relacionados a quadros ansiosos. Embora alguns estudos tenham demonstrado que o comportamento inibido na infância estaria ligado ao TP na vida, essa associação é ainda controversa, e esse fenótipo intermediário parece estar associado consistentemente com o transtorno de ansiedade social. A sensibilidade à ansiedade na infância parece ser um fenótipo específico do TP; no entanto, ela é responsável pela explicação de apenas uma pequena parte dos casos. Neuroticismo e evitação de danos estão também elevados nos pacientes com TP, mas eles são fenótipos inespecíficos, ligados a outros transtornos de ansiedade e a transtornos de humor ${ }^{25,26}$.

Diversos polimorfismos em genes candidatos foram investigados para associação com o TP e com os fenótipos intermediários ligados a ele, mas nenhum dos achados foi consistentemente replicado por estudos posteriores, não havendo evidências contundentes de algum gene especificamente ligado ao TP com uma magnitude que represente alguma importância clínica. Cabe assinalar que além de se dedicar ao estudo das heranças de genes específicos conferindo risco para psicopatologia psiquiátrica, a psiquiatria atual tem se voltado para o estudo da epigenética, isto é, o estudo de processos celulares chaves que integram diversos estímulos ambientais para exercer mudanças potentes e, muitas vezes, de longa duração na expressão gênica por meio da regulação da estrutura da cromatina, ou seja, "escolhendo o que irá ser codificado do código genético"27. Espera-se que os avanços em estudos de epigenética sejam a chave para o entendimento de diversos transtornos com etiologia multifatorial e complexa como o TP.

Alguns estudos de neuroimagem estrutural encontraram algumas diferenças entre os cérebros de pacientes com TP e os de controles. Um volume reduzido nos lobos temporais e na amígdala foi encontrado entre pacientes com TP. Outros estudos detectaram uma redução de massa cinzenta no giro parahipocampal esquerdo, redução bilateral na massa cinzenta do putâmen e um aumento no tronco cerebral, especificamente nos sítios rostrais, na ínsula esquerda e giro temporal superior esquerdo, mesencéfalo e ponte ${ }^{28}$. Os estudos de neuroimagem funcional são pouco conclusivos em virtude da dificuldade de encontrar paradigmas válidos para esse transtorno.

Considerando as bases teóricas para o entendimento do TP do ponto de vista psicológico, uma das teorias mais aceitas e com grande utilidade prática segue a teoria cognitivo-comportamental. Segundo Clark ${ }^{29}$, os ataques de pânico derivam de interpretações catastróficas disfuncionais de certas manifestações corporais. A suposição é centralizada no processamento inadequado de informações vindas de um estímulo externo (ruído, luminosidade) ou interno (sensação de taquicardia, sudorese, vertigem). A interpretação é de perigo iminente, o que dispara ou intensifica as sensações corporais, confirmando o "perigo" e gerando, então, mais interpretações catastróficas e ansiedade em uma espiral crescente e rápida. $\mathrm{O}$ modelo de Barlow $^{30}$ amplia este conceito. Conforme este modelo, o ataque de pânico inicial é um alarme falso que pode ser ativado quando ocorre um aumento momentâneo de estresse na vida de indivíduos com vulnerabilidades biológicas e/ou psicológicas. Após o primeiro ataque, a pessoa torna-se apreensiva em relação a ataques futuros. Para Barlow ${ }^{30}$, o medo primário no TP é o medo das sensações físicas, particularmente as associadas à ativação autonômica, com influência de fatores socioculturais para o surgimento da agorafobia.

Outros autores dedicaram-se ao estudo dos fatores psicodinâmicos associados ao início do TP ou ao de uma maior vulnerabilidade psicológica ao transtorno ${ }^{31}$. Pacientes com TP frequentemente se descrevem como crianças medrosas, nervosas e tímidas, e também referem desconforto com os sentimentos agressivos, sentimentos crônicos de baixa autoestima, frustração e ressentimento precedentes ao início do TP. A qualidade da relação parental é um fator de risco para o TP ${ }^{32}$. Por exemplo, os pacientes com TP frequentemente descrevem seus pais como pessoas brabas, críticas, amedrontadoras e controladoras ${ }^{33}$. A 
teoria psicodinâmica trabalha com a prerrogativa de que conflitos internos inconscientes se relacionam estreitamente com as manifestações ansiosas; portanto, as frustrações, ressentimentos e relações infantis tornam-se imperiosas para a abordagem terapêutica desse transtorno, assim como uma cuidadosa avaliação da estrutura de personalidade subjacente do indivíduo.

As bases biológicas, sejam elas genéticas ou neuroanatômicas, e as teorias psicológicas, tanto cognitivo-comportamentais quanto psicodinâmicas, não são estanques: elas estão em constante atualização e evolução conceitual e, ao contrário do que possa se pensar, não são excludentes e sim complementares. Um transtorno complexo como o TP só pode ser bem compreendido com um arcabouço teórico extenso que englobe aspectos de diversas linhas de pensamento.

\section{Diagnóstico}

O TP está classificado no Diagnostic and Statistical Manual of Mental Disorders, Fourth Edition, Text Revision (DSM-IV$\mathrm{TR})^{34}$, dentro do grupo dos transtornos de ansiedade, e os critérios definidos pelo DSM-IV-TR são muito semelhantes aos encontrados na Classificação Internacional de Doenças 10 (CID-10).

O diagnóstico do TP é essencialmente clínico. Os critérios diagnósticos para TP segundo o DSM-IV-TR são apresentados na Tabela 1.

Diversos autores propuseram subtipos de TP baseados na apresentação sintomática do ataque (tipo cardiorrespiratório, autonômico/somático, cognitivo), período do dia em que $\mathrm{o}$ ataque ocorre (diurno, noturno), idade de início (precoce e tardio), curso (limitado, crônico), etc. No entanto, ainda não há consistência na definição desses subtipos, especialmente pelo manejo semelhante do ponto de vista terapêutico ${ }^{24}$.

\section{Diagnóstico diferencial}

Um dos pontos chaves na avaliação dos pacientes com ataques de pânico é o diagnóstico diferencial, especialmente pela interface que essa apresentação tem com outros problemas clínicos de saúde. Assim, embora o diagnóstico do TP seja essencialmente clínico, uma série de situações clínicas e psiquiátricas têm apresentações que incluem ataques de pânico ou que se assemelham a ele. Assim, o algoritmo de avaliação dos ataques de pânico inclui: ataques secundários a uma condição clínica (por exemplo, hipertireoidismo, feocromocitoma), ao uso ou abstinência de substâncias (por exemplo, abuso de cocaína, abstinência de álcool), a transtornos de ansiedade (como o TP) e a outros transtornos psiquiátricos.

Em virtude do caráter agudo do ataque de pânico, seu diagnóstico diferencial é essencial nas emergências médicas e deve ser guiado segundo a apresentação clínica. A avaliação de doenças clínicas de acordo com a sintomatologia apresentada é essencial para um bom manejo dos ataques. O abuso de estimulantes, bem como a abstinência de depressores do sistema nervoso central, deve ser avaliado na anamnese como possível responsável dos ataques. Uma história detalhada das medicações e drogas em uso pelo paciente deve ser colhida, especialmente a história de consumo de álcool, cafeína, cocaína, nicotina, broncodilatadores e hipnóticos sedativos. Caso o ataque não possa ser mais bem explicado por nenhuma condição clínica ou pelo uso ou abstinência de substâncias, deve-se proceder à investigação diagnóstica para transtornos psiquiátricos, com atenção especial para os transtornos de ansiedade e, principalmente, para o TP. Um algoritmo para avaliação diagnóstica dos ataques é apresentado na Figura 1.

Tabela 1 - Critérios diagnósticos para transtorno do pânico segundo o Diagnostic and Statistical Manual of Mental Disorders, Fourth Edition, Text Revision

A. São necessários os critérios 1 e 2:

1. Ataques de pânico recorrentes e espontâneos (inesperados).

2. Pelo menos um ataque foi seguido, durante um mês ou mais, das seguintes características:

a) preocupação persistente em relação a ataques adicionais;

b) preocupação em relação às implicações do ataque ou às suas consequências (perder o controle, ter um ataque cardíaco, enlouquecer, etc.);

c) alteração significativa do comportamento relacionada às crises de pânico.

B. Deve-se especificar se há ou não agorafobia associada.

C. Os ataques de pânico não se devem aos efeitos fisiológicos diretos de uma substância (por exemplo, abuso de droga ou medicamento) ou a uma condição médica geral (por exemplo, hipertireoidismo).

D. Os ataques de pânico não são mais bem explicados por outro transtorno mental como fobia social (que ocorre, por exemplo, em situações de exposição a eventos sociais, como falar em público), fobia específica (por exemplo, na presença de um animal específico), transtorno obsessivo compulsivo (por exemplo, quando exposto à sujeira), transtorno de estresse pós-traumático ou transtorno de ansiedade de separação (por exemplo, em crianças, em resposta a estar afastado do lar). 


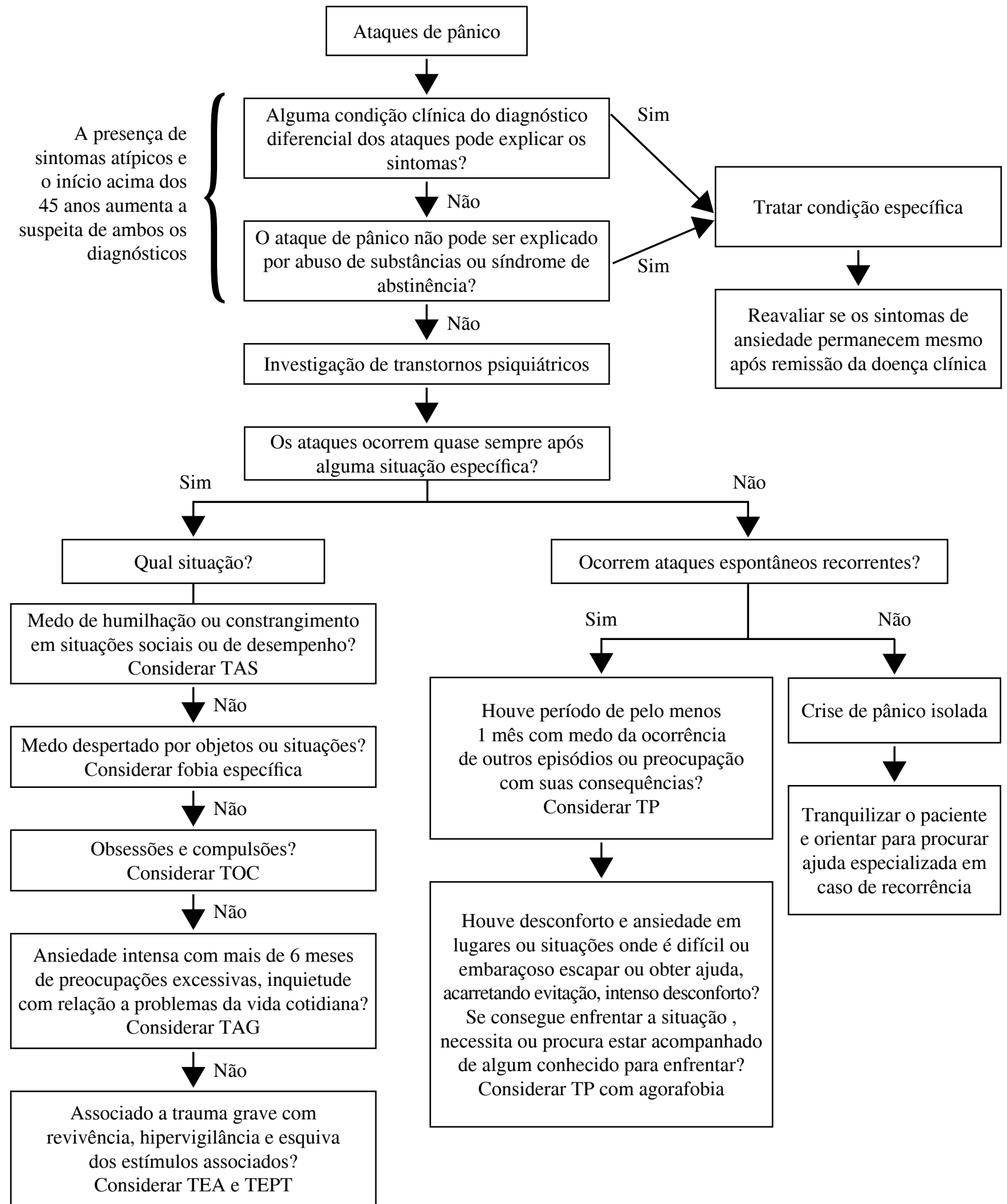

TAS $=$ transtorno de ansiedade social; TOC $=$ transtorno obsessivo compulsivo; TAG $=$ transtorno de ansiedade generalizada; TEA $=$ transtorno de estresse agudo; TEPT = transtorno de estresse pós-traumático; TP = transtorno do pânico.

Figura 1 - Algoritmo de avaliação diagnóstica dos ataques de pânico 


\section{Tratamento}

\section{Manejo dos ataques de pânico}

O manejo emergencial das crises de pânico baseia-se principalmente na tranquilização do paciente mediante a informação de que os seus sintomas são provenientes de um ataque de ansiedade, não configurando uma condição clínica grave com risco de morte iminente (no caso de ataques relacionados a transtornos psiquiátricos), no reforço de que a crise é realmente intensa, muito desagradável e causa mal-estar muito forte. Deve-se reforçar o caráter passageiro (cerca de 10-30 minutos) do ataque e, especialmente, instruir ao paciente para que ele respire pelo nariz e não pela boca, enfatizando a importância de ele tentar controlar a frequência de inspirações no intuito de não hiperventilar. Em grande parte das vezes, a tranquilização rápida e o caráter autolimitado dos sintomas são suficientes para terminar com a crise. Nos pacientes com sintomas predominantemente respiratórios, relacionados provavelmente a hiperventilação, o paciente é instruído a respirar com o diafragma e limitar o uso da musculatura intercostal. Deve-se estimulá-lo a respirar lentamente até que os sintomas de hiperventilação desapareçam. Algumas técnicas de relaxamento também podem ser utilizadas. Por exemplo, pode-se instruir o paciente a permanecer deitado, com os olhos fechados, respirando lenta e profundamente, tentando relaxar os diferentes grupos musculares e concentrando-se em um cenário tranquilo. No entanto, se a crise for muito intensa ou de tempo prolongado, o uso de psicofármacos pode ser aconselhado. Os benzodiazepínicos de ação curta são a primeira escolha nesses casos e, apesar de carecerem de evidências que suportem afirmações mais encorajadoras, esses psicofármacos são bastante utilizados na prática clínica. Há algumas evidências da utilidade do uso de benzodiazepínicos especialmente na dor torácica cardíaca e não cardíaca, mostrando que essa medicação diminui a ansiedade, a dor e a ativação cardiovascular. Nestes estudos, os benzodiazepínicos mostraram-se seguros ao serem administrados isoladamente ou em conjunto com outras medicações ${ }^{35}$. Um algoritmo para essa avaliação e manejo é apresentado na Figura 2.

\section{Tratamento do transtorno do pânico com ou sem agorafobia}

O tratamento precoce do TP é essencial no sentido de reduzir o sofrimento e prejuízo associado ao transtorno e no intuito de prevenir o surgimento de complicações e comorbidades, além dos custos sociais do transtorno. Além disso, tratar o TP reduz significativamente os gastos sociais, tendo em vista que, embora aumente os gastos diretos com consultas e medicações psiquiátricas, há uma redução importante nas visitas aos serviços de emergência e nas consultas médicas não psiquiátricas ${ }^{36}$.

A prevenção de novas crises e a diminuição das complicações associadas a elas, como a ansiedade antecipatória e a evitação fóbica, são os pontos chaves no tratamento do TP. Deve-se dar especial atenção para as comorbidades como os transtornos de humor e o uso de substâncias. De forma geral, existem três formas de tratamento do TP: o tratamento psicofarmacológico, o psicoterapêutico e o combinado.

\section{Psicofármacos}

Os graus de evidência de cada recomendação serão marcados pelos símbolos: A Grau de recomendação A (recomendação bem estabelecida - proveniente de mais de um ensaio clínico randomizado de boa qualidade); ${ }^{\mathrm{B}}$ Grau de recomendação B (recomendação estabelecida, mas sujeita a novas investigações - proveniente de um ensaio clínico randomizado ainda não replicado); ${ }^{\mathrm{C}}$ Grau de recomendação C (recomendação fraca - proveniente de estudos não controlados ${ }^{\mathrm{Cl}}$, de relatos de caso e de opinião de especialista $\left.{ }^{\mathrm{C} 2}\right) ; \mathrm{e}^{\mathrm{D}}$ Grau de recomendação $\mathrm{D}$ (recomendação inconsistente - com evidência em número semelhante mostrando ineficácia e benefício da medicação) ${ }^{37}$.

Dentre os psicofármacos, os inibidores seletivos da recaptação da serotonina (ISRS) (fluoxetina ${ }^{\mathrm{A}}$, sertralina ${ }^{\mathrm{A}}$, paroxetina $^{\mathrm{A}}$, fluvoxamina ${ }^{\mathrm{A}}$, citalopram $^{\mathrm{A}}$ e escitaloram ${ }^{\mathrm{A}}$ ) e a venlafaxina $^{\mathrm{A}}$, um inibidor de recaptação da serotonina e da noradrenalina (IRSN), constituem-se a primeira escolha farmacológica para o TP. Os tricíclicos (clomipramina ${ }^{\mathrm{A}}$ e imipramina $^{\mathrm{A}}$ ) são igualmente eficazes, mas são menos tolerados que os ISRS e podem ser letais em superdose; por esses motivos, eles podem ser utilizados como segunda escolha no tratamento do TP. Não há diferença de eficácia entre os agentes de primeira linha previamente apresentados ${ }^{37-40}$. O uso de benzodiazepínicos $\left(\right.$ alprazolam $^{\mathrm{A}}$, clonazepam $^{\mathrm{A}}$, diazepam ${ }^{\mathrm{A}}$ e lorazepam ${ }^{A}$ ) é controverso, sendo que algumas diretrizes internacionais recomendam seu uso para casos refratários em pacientes sem história de dependência e permitem o uso concomitante nas primeiras semanas de uso dos ISRS tendo em vista sua eficácia em curto prazo - 1 dia a 1 semana $^{37-39}$ -, enquanto outras não recomendam seu uso ${ }^{40}$ pelo risco da dependência. Na prática clínica, seu uso é corrente e pode ajudar no manejo dos pacientes com TP. No entanto, deve-se atentar para o risco de dependência durante todo o tratamento em qualquer paciente ${ }^{37}$.

Alguns estudos apontam para a eficácia de uso dos inibidores da monoaminoxidase ${ }^{\mathrm{B}}$ (IMAO), mas deve-se atentar para a possibilidade de sérios efeitos adversos devido a interações com outras drogas e componentes alimentares. A fenelzina, um IMAO, só deve ser prescrita após falha de agentes de primeira linha, ou intolerância às medicações previamente citadas. Dentre outras medicações que evidenciaram eficácia no TP estão: ondansetrona ${ }^{\mathrm{B}}$, bupropiona ${ }^{\mathrm{B}}$, tiagabina $^{\mathrm{B}}$, vigabatrina ${ }^{\mathrm{B}}$, milnaciprano ${ }^{\mathrm{B}}$, combinações de ISRS com tricíclicos ${ }^{\mathrm{B}}$, olanzapina em monoterapia ${ }^{\mathrm{B}}$, adição de olanzapina a um ISRS ${ }^{\mathrm{B}}$, adição de pindolol a um ISRS $^{\mathrm{B}}$ e a combinação de valproato e clonazepam ${ }^{\mathrm{B}}{ }^{37}$. Além disso, há evidências preliminares que também suportam o uso da duloxetina $^{\mathrm{C}}$, um outro IRSN, para o $\mathrm{TP}^{41}$. 


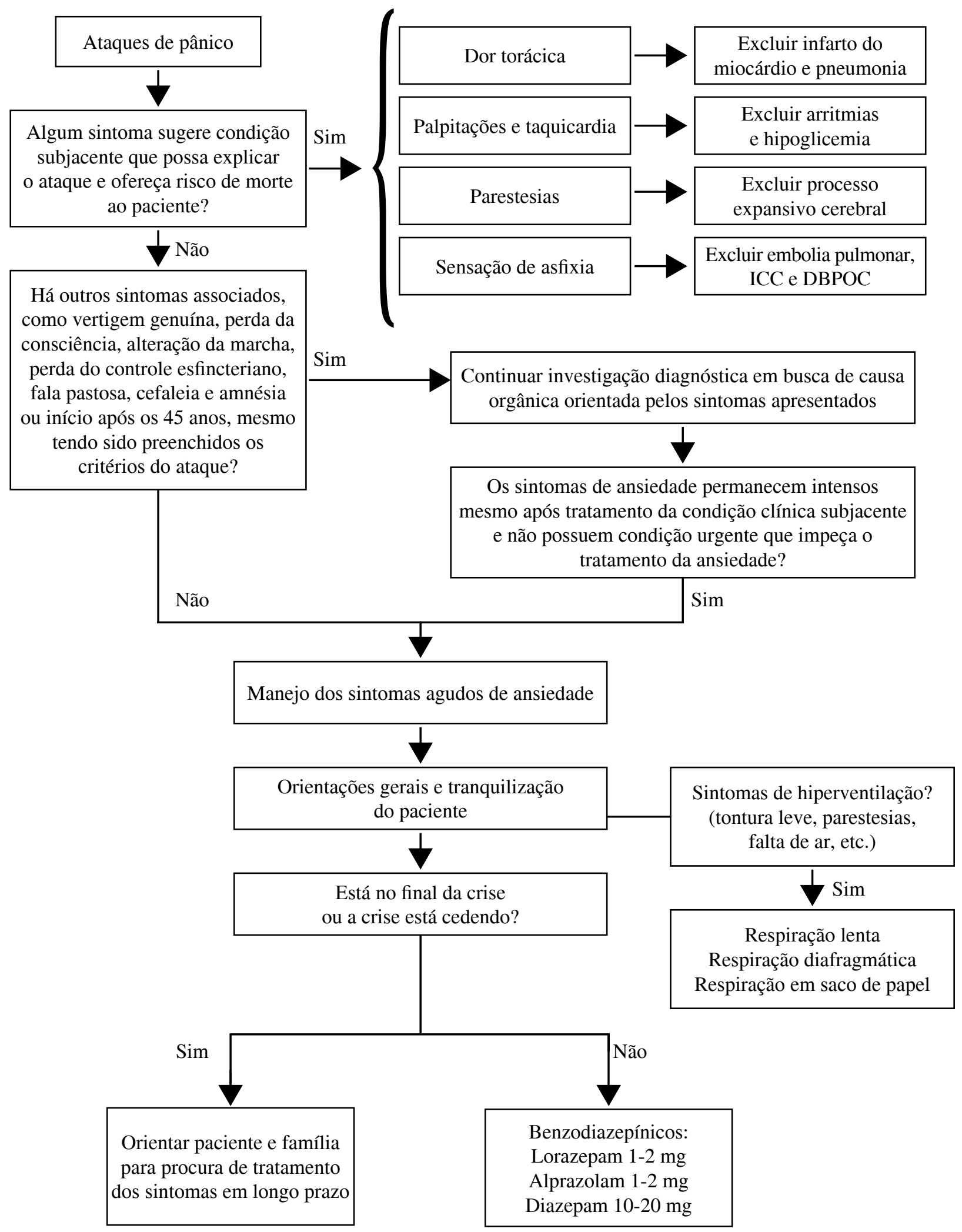

ICC $=$ insuficiência cardíaca congestiva; DBPOC $=$ doença bronco-pulmonar obstrutiva crônica.

Figura 2 - Algoritmo de manejo emergencial dos ataques de pânico 
Em casos resistentes, olanzapina em monoterapia ${ }^{\mathrm{C} 1}$, adição de fluoxetina a um tricíclico ${ }^{\mathrm{Cl}}$, adição de tricíclico à fluoxetina ${ }^{\mathrm{Cl}}$, adição de olanzapina a um ISRS ${ }^{\mathrm{Cl}}$ podem ser utilizados de acordo com estudos abertos. Adição de lítio à clomiprami$\mathrm{na}^{\mathrm{C} 2}$, ou uma combinação de clonazepam com valproato ${ }^{\mathrm{C} 2}$ foram eficazes de acordo com relatos de caso. A moclobemida ${ }^{\mathrm{D}}$ e reboxetina ${ }^{\mathrm{D}}$ apresentaram resultados inconsistentes ${ }^{37}$.

Um período de 12 semanas é o tempo esperado de ponto de corte para se tentar uma nova alternativa terapêutica e considerar a primeira uma falha. Alguns autores sugerem que uma resposta menor do que $25 \%$ nos sintomas nas primeiras 8 semanas de tratamento já são suficientes para uma segunda tentativa. No entanto, as decisões clínicas devem ser individualizadas com base em experiência clínica, tendo em vista a falta de evidências nessa área. As diretrizes recomendam que o tratamento de manutenção se dê por cerca de 1 a $2 \operatorname{anos}^{37}$.

\section{Psicoterapias}

A terapia cognitivo-comportamental (TCC) é a terapia com os resultados mais consistentes para o TP, sendo superior a terapias de controle de atenção psicossocial e a placebo na maioria dos estudos e apresentando uma boa aceitabilidade e aderência, rápido início de ação e uma boa relação de custo-efetividade ${ }^{42}$. Tanto a TCC em grupo quanto individual possuem resultados semelhantes. Duas grandes meta-análises relataram grandes tamanhos de efeito de 1,55 (resposta de $63 \%)^{43}$ e de $0,90^{44}$ para TCC para TP. A TCC é também uma estratégia de resgate de pacientes com má resposta prévia às medicações e de relativa efetividade para as condições comórbidas do $\mathrm{TP}^{37-40}$. Estudos recentes têm pesquisado a eficácia da TCC por computador e internet, também mostrando resultados promissores e comparáveis aos da terapia padrão ${ }^{45,46}$.

O tratamento com TCC para TP consiste em psicoeducação sobre o TP, no intuito de corrigir interpretações errôneas acerca do TP, treinamento de técnicas para diminuir a ansiedade, como respiração diafragmática e relaxamento muscular, reestruturação cognitiva, para identificar e corrigir distorções no pensamento, exposição interoceptiva, no intuito de que o paciente aprenda a lidar com os sintomas físicos do ataque de pânico, e exposição in vivo, a fim de estimulálo a enfrentar as principais situações que teme por medo de passar mal e não encontrar saída ou ajuda.

Há evidências preliminares de eficácia de tratamentos breves de base psicodinâmica focados para o $\mathrm{TP}^{47}$. Embora com poucas evidências, o tratamento psicanalítico e a psicoterapia de orientação psicanalítica são muito utilizados na prática clínica em determinados centros ${ }^{37}$. Dentre os motivos para a falta de evidências encontram-se: o formato de longa duração, o foco em outros desfechos que não estritamente nos sintomas e nos diagnósticos estruturados do DSM-IV-TR. A despeito disso, esse tipo de psicoterapia parece oferecer resultados duradouros, com menores taxas de recaídas e vantagens em desfechos não convencionais, como o uso de estilos defensivos mais maduros, melhora nas relações interpessoais e nos conflitos intrapsíquicos e, consequentemente, uma melhor qualidade de vida em termos gerais de funcionamento psicossocial.

\section{Terapias combinadas}

Uma meta-análise envolvendo cerca de 21 ensaios clínicos randomizados que incluíram mais de 1.700 pacientes com TP com ou sem agorafobia claramente mostrou que o tratamento combinado de antidepressivos e psicoterapia foi mais efetivo que as duas alternativas terapêuticas em monoterapia na fase aguda do transtorno. Após a descontinuação, os pacientes que receberam tratamento combinado continuaram a se beneficiar em relação àqueles que receberam apenas medicação, mas não houve diferenças com respeito àqueles que receberam apenas psicoterapia, com uma aparente vantagem para as técnicas baseadas nos preceitos cognitivocomportamentais $^{48}$. Portanto, embora haja pouca evidência comparando modalidades terapêuticas para o TP, a inclusão de terapia cognitiva em algum momento do tratamento provavelmente melhore desfechos em longo prazo ${ }^{24}$.

Da perspectiva da saúde pública, algumas diretrizes ${ }^{37,39}$ preconizam que ao menos duas tentativas com tratamento de primeira linha devem ser tentadas com um médico generalista antes de encaminhamento para um psiquiatra especializado. Obviamente, essa indicação deve ser individualizada, tendo em vista as nuances da prática clínica e as peculiaridades da prática médica em cada região.

\section{Prognóstico e prevenção}

Para uma grande parcela dos pacientes, o TP tem o prognóstico de um transtorno crônico com recaídas e agudizações frequentes, em geral associadas aos eventos estressores de vida, e possui fases de remissão parcial ou completa dos sintomas. Vários estudos mostraram claramente que a descontinuação de medicações resulta em recaída em um substancial número de pacientes, com taxas de cerca de $25-50 \%$, dentro de 6 meses. Embora se pense que as taxas sejam menores para a TCC, um grande número de pacientes apresenta recaída também com essas alternativas terapêuticas, e isso faz com que o TP seja considerado um transtorno de curso crônico para um grande número de pacientes. De forma adicional, os ISRS, tricíclicos, IRSN e benzodiazepínicos são associados com síndrome de retirada (muito pior para benzodiazepínicos), que pode ser um estímulo interoceptivo promotor da recaída do TP e favorecedor da cronicidade.

Em virtude da cronicidade dos transtornos mentais, a psiquiatria tem se voltado para estudar estratégias de prevenção. Algumas estratégias recentes encontraram resultados promissores com protocolos de terapia em sujeitos com sintomas subclínicos do transtorno ${ }^{49}$. Além disso, é sabido que um dos principais fatores de risco para transtornos de ansiedade na vida adulta é a presença de transtornos ou traços de ansiedade durante a infância e a adolescência ${ }^{26}$. Nesse sentido, de forma 
cada vez mais precoce, pesquisas atuais vêm se focando no tratamento precoce dos transtornos de ansiedade ou mesmo na prevenção em crianças de risco, como os filhos de pais com transtornos de ansiedade, por exemplo. Há poucas pesquisas investigando a eficácia dessas estratégias de prevenção; no entanto, este é um campo promissor de pesquisas futuras.

\section{Referências}

1. Simpson RJ, Kazmierczak T, Power KG, Sharp DM. Controlled comparison of the characteristics of patients with panic disorder. Br J Gen Pract. 1994;44(385):352-6.

2. Ramage-Morin PL. Panic disorder and coping. Health Rep. 2004;15 Suppl:31-43.

3. Roy-Byrne PP, Stein MB, Russo J, Mercier E, Thomas R, McQuaid J, et al. Panic disorder in the primary care setting: comorbidity, disability, service utilization, and treatment. J Clin Psychiatry. 1999;60(7):492-9; quiz 500.

4. Goodwin RD, Roy-Byrne P. Panic and suicidal ideation and suicide attempts: results from the National Comorbidity Survey. Depress Anxiety. 2006;23(3):124-132.

5. Sareen J, Cox BJ, Afifi TO, de Graaf R, Asmundson GJ, ten Have M, et al. Anxiety disorders and risk for suicidal ideation and suicide attempts: a population-based longitudinal study of adults. Arch Gen Psychiatry. 2005;62(11):1249-57.

6. Smoller JW, Pollack MH, Wassertheil-Smoller S, Jackson RD, Oberman A, Wong ND, et al. Panic attacks and risk of incident cardiovascular events among postmenopausal women in the Women's Health Initiative Observational Study. Arch Gen Psychiatry. 2007;64(10):1153-60.

7. Bull Bringager C, Arnesen H, Friis S, Husebye T, Dammen T. A long-term followup study of chest pain patients: effect of panic disorder on mortality, morbidity, and quality of life. Cardiology. 2008;110(1):8-14.

8. American Psychiatric Association. Diagnostic and statistical manual of mental disorders. Washington, DC: APA; 1980.

9. Wooley CF. Jacob Mendez Da Costa: medical teacher, clinician, and clinical investigator. Am J Cardiol. 1982;50(5):1145-8.

10. Wheeler EO, White PD, et al. Neurocirculatory asthenia, anxiety neurosis, effort syndrome, neurasthenia; a 20 year follow-up study of 173 patients. J Am Med Assoc. 1950;142(12):878-89.

11. Nixon PG. The grey area of effort syndrome and hyperventilation: from Thomas Lewis to today. J R Coll Physicians Lond. 1993;27(4):377-83.

12. Kessler RC, Chiu WT, Jin R, Ruscio AM, Shear K, Walters EE. The epidemiology of panic attacks, panic disorder, and agoraphobia in the National Comorbidity Survey Replication. Arch Gen Psychiatry. 2006;63(4):415-24.

13. Mari JJ, Jorge MR, Kohn R. Epidemiologia dos transtornos psiquiátricos em adultos. In: de Mello MF, de Mello AA, Kohn R, eds. Epidemiologia da Saúde Mental no Brasil. Porto Alegre: Artmed; 2006. p. 119-42.

14. Rosenberg R, Ottosson JO, Bech P, Mellergård M, Rosenberg NK. Validation criteria for panic disorder as a nosological entity. Acta Psychiatr Scand Suppl. 1991;365:7-17.

15. Rothe C, Koszycki D, Bradwejn J, King N, De Luca V, Shaikh S, et al. Association study of serotonin-2A receptor gene polymorphism and panic disorder in patients from Canada and Germany. Neurosci Lett. Jun 17 2004;363(3):276-9.

16. Hettema JM, Neale MC, Kendler KS. A review and meta-analysis of the genetic epidemiology of anxiety disorders. Am J Psychiatry. 2001;158(10):1568-78.

17. Crowe RR, Noyes R, Pauls DL, Slymen D. A family study of panic disorder. Arch Gen Psychiatry. 1983;40(10):1065-9.

18. Fergusson DM, Lynskey MT, Horwood LJ. Childhood sexual abuse and psychiatric disorder in young adulthood: I. Prevalence of sexual abuse and factors associated with sexual abuse. J Am Acad Child Adolesc Psychiatry. 1996;35(10):1355-64.

19. Kendler KS, Bulik CM, Silberg J, Hettema JM, Myers J, Prescott CA. Childhood sexual abuse and adult psychiatric and substance use disorders in women: an epidemiological and cotwin control analysis. Arch Gen Psychiatry. 2000;57(10):953-9.

20. Kendler KS, Neale MC, Kessler RC, Heath AC, Eaves LJ. Childhood parental loss and adult psychopathology in women. A twin study perspective. Arch Gen Psychiatry. 1992;49(2):109-16.

21. Hettema JM, Prescott CA, Myers JM, Neale MC, Kendler KS. The structure of genetic and environmental risk factors for anxiety disorders in men and women. Arch Gen Psychiatry. 2005;62(2):182-9.

22. Faravelli C. Life events preceding the onset of panic disorder. J Affect Disord. 1985;9(1):103-5.

23. Scocco P, Barbieri I, Frank E. Interpersonal problem areas and onset of panic disorder. Psychopathology. 2007;40(1):8-13.
24. Roy-Byrne PP, Craske MG, Stein MB. Panic disorder. Lancet. 2006;368(9540):1023-32 .

25. Hirshfeld-Becker DR, Micco J, Henin A, Bloomfield A, Biederman J, Rosenbaum J. Behavioral inhibition. Depress Anxiety. 2008;25(4):357-67.

26. Hirshfeld-Becker DR, Micco JA, Simoes NA, Henin A. High risk studies and developmental antecedents of anxiety disorders. Am J Med Genet C Semin Med Genet. 2008;148C(2):99-117.

27. Nestler EJ. Epigenetic mechanisms in psychiatry. Biol Psychiatry. 2009;65(3):189-90.

28. Ferrari MC, Busatto GF, McGuire PK, Crippa JA. Structural magnetic resonance imaging in anxiety disorders: an update of research findings. Rev Bras Psiquiatr. 2008;30(3):251-64

29. Clark DM. A cognitive approach to panic. Behav Res Ther. 1986;24(4):461-70.

30. Barlow DH. Anxiety and its disorders: The nature and treatment of anxiety and panic. New York: Guilford Press; 1988.

31. Kipper L, Blaya C, Teruchkin B, Heldt E, Isolan L, Mezzomo K, et al. Evaluation of defense mechanisms in adult patients with panic disorder: before and after treatment. J Nerv Ment Dis. 2005;193(9):619-24.

32. Heider D, Matschinger H, Bernert S, Alonso J, Brugha TS, Bruffaerts R, et al. Adverse parenting as a risk factor in the occurrence of anxiety disorders: A study in six European countries. Soc Psychiatry Psychiatr Epidemiol. 2008;43(4):266-72.

33. Shear MK, Cooper AM, Klerman GL, Busch FN, Shapiro T. A psychodynamic model of panic disorder. Am J Psychiatry. 1993;150(6):859-66.

34. American Psychiatric Association. Diagnostic and Statistical Manual of Mental Disorders, Fourth Edition, Text Revision. Washington, DC: APA; 1994.

35. Manfro GG, Blaya C, Salum GA. Ansiedade aguda: Ataques de pânico. In: Quevedo J, Schmitt R, Kapczinski F, eds. Emergência psiquiátricas. Segunda ed. Porto Alegre: ARTMED; 2008. p. 197-217.

36. Lin HC, Chen SF, Chen YH, Lee HC. Healthcare utilization patterns before and after contact with psychiatrist care for panic disorder. J Affect Disord. Mar 92009. In press.

37. Bandelow B, Zohar J, Hollander E, Kasper S, Möller HJ, WFSBP Task Force on Treatment Guidelines for Anxiety, Obsessive-Compulsive and Post-Traumatic Stress Disoders, et al. World Federation of Societies of Biological Psychiatry (WFSBP) guidelines for the pharmacological treatment of anxiety, obsessivecompulsive and post-traumatic stress disorders - first revision. World J Biol Psychiatry. 2008;9(4):248-312.

38. Canadian Psychiatric Association. Clinical practice guidelines. Management of anxiety disorders. Can J Psychiatry. 2006;51(8 Suppl 2):9S-91S.

39. Baldwin DS, Anderson IM, Nutt DJ, Bandelow B, Bond A, Davidson JR, et al. Evidence-based guidelines for the pharmacological treatment of anxiety disorders: recommendations from the British Association for Psychopharmacology. J Psychopharmacol. 2005;19(6):567-96.

40. McIntosh A, Cohen A, Turnbull N, Esmonde L, Dennis P, Eatock J, et al. Clinical Guidelines and Evidence Review for Panic Disorder and Generalised Anxiety Disorder. Sheffield: University of Sheffield/London - National Collaborating Centre for Primary Care; 2004

41. Simon NM, Kaufman RE, Hoge EA, Worthington JJ, Herlands NN, Owens ME, et al. Open-label support for duloxetine for the treatment of panic disorder. CNS Neurosci Ther. 2009;15(1):19-23.

42. Manfro GG, Heldt E, Cordioli AV, Otto MW. [Cognitive-behavioral therapy in panic disorder]. Rev Bras Psiquiatr. 2008;30 Suppl 2:s81-7.

43. Mitte K. A meta-analysis of the efficacy of psycho- and pharmacotherapy in panic disorder with and without agoraphobia. J Affect Disord. 2005;88(1):27-45.

44. Westen D, Morrison K. A multidimensional meta-analysis of treatments for depression, panic, and generalized anxiety disorder: an empirical examination of the status of empirically supported therapies. J Consult Clin Psychol. 2001;69(6):875-99.

45. Kiropoulos LA, Klein B, Austin DW, Gilson K, Pier C, Mitchell J, et al. Is internet-based CBT for panic disorder and agoraphobia as effective as face-toface CBT? J Anxiety Disord. 2008;22(8):1273-84.

46. Klein B, Austin D, Pier C, Kiropoulos L, Shandley K, Mitchell J, et al. Internetbased treatment for panic disorder: does frequency of therapist contact make a difference? Cogn Behav Ther. 2009:1-14. In press.

47. Milrod B, Leon AC, Busch F, Rudden M, Schwalberg M, Clarkin J, et al. A randomized controlled clinical trial of psychoanalytic psychotherapy for panic disorder. Am J Psychiatry. 2007;164(2):265-72

48. Furukawa TA, Watanabe N, Churchill R. Psychotherapy plus antidepressant for panic disorder with or without agoraphobia: systematic review. Br J Psychiatry. 2006;188:305-12.

49. Meulenbeek P, Willemse G, Smit F, Smits N, van Balkom A, Spinhoven P, et al. Effects and feasibility of a preventive intervention in sub-threshold and mild panic disorder: Results of a pilot study. BMC Res Notes. 2009;2:4. 